activate IFN downstream of nucleic acid sensors may help to define subpopulations of SLE.

Funding Source(s): National Council for Scientific and Technological Development (CNPq 157534/2015-4)

PBMCs were prepared from fresh healthy human blood, stimulated with individual patient SLE serum in combination with defined antigens corresponding to their ENA or ANA titer profile. After 20 hour, IFNalpha protein levels were quantified and expressed relative to agonist ssRNA40. Data shown are from 2 more independent donors with total SLE patients numbers indicated in parenthesis. $* * * \mathrm{p}<0.0001$ (unpaired t-test).

\section{IMMUNOSUPPRESSANT DISCONTINUATION IN SYSTEMIC LUPUS ERYTHEMATOSUS: PREVALENCE, RISK OF SUBSEQUENT FLARE AND EFFECT ON DAMAGE ACCRUAL}

Margherita Zen*, Francesca Saccon, Maddalena Larosa, Mariele Gatto, Luca laccarino, Andrea Doria. University of Padova, Department of Medicine, Division of Rheumatology

10.1136/lupus-2019-Ism.173

Background Patients with Systemic Lupus Erythematosus (SLE) are often exposed to prolonged treatment with immunosuppressants (IS). Remission of disease activity should conceptually

Abstract 173 Table 1 Characteristics of remitted patients who discontinued IS, overall and according to the occurrence of a subsequent disease flare. Data refer to mean \pm SD

\begin{tabular}{|c|c|c|c|c|}
\hline & $\underset{(105)}{\text { Remitted pts }}$ & $\begin{array}{l}\text { Patients with } \\
\text { flare (26) }\end{array}$ & $\begin{array}{l}\text { Patients without } \\
\text { flare (79) }\end{array}$ & $\mathrm{P}$ values \\
\hline Female, N (\%) & $93(88.6)$ & $22(84.6)$ & $71(89.9)$ & n.s. \\
\hline Age at SLE onset, years & $25 \pm 9$ & $22 \pm 8$ & $26 \pm 9$ & 0.028 \\
\hline Age at 2018, years & $44 \pm 11$ & $40 \pm 11$ & $45 \pm 11$ & 0.035 \\
\hline SLE duration at 2018 , years & $19.5 \pm 9.2$ & $18.3 \pm 8.7$ & $19.1 \pm 9.4$ & n.s. \\
\hline SDI at 2017 & $1.1 \pm 1.48$ & $0.96 \pm 1.50$ & $1.15 \pm 1.50$ & n.s. \\
\hline SLE duration at IS discontinuation, years & $10.3 \pm 8.2$ & $8.8 \pm 6.4$ & $11.2 \pm 8.6$ & n.s. \\
\hline SLE duration at remission, years & $5.2 \pm 6.1$ & $4.3 \pm 4.2$ & $6.4 \pm 7.4$ & n.s. \\
\hline $\begin{array}{l}\text { Remission duration at IS discontinuation, } \\
\text { months }\end{array}$ & $42 \pm 29$ & $28 \pm 16$ & $46 \pm 31$ & $<0.001$ \\
\hline Time to achieve remission, months & $32 \pm 43$ & $26 \pm 35$ & $34 \pm 45$ & n.s. \\
\hline Complete remission, N $(\%)$ & $17(16.2)$ & $4(15.4)$ & $13(16.5)$ & n.s. \\
\hline $\begin{array}{l}\text { HCQ maintenance therapy after IS } \\
\text { discontinuation, } N(\%)\end{array}$ & $83(79)$ & $16(61.5)$ & $67(84.8)$ & 0.015 \\
\hline \multicolumn{5}{|l|}{ Reason for IS therapy } \\
\hline Lupus Nephritis, N (\%) & $68(64.8)$ & $14(53.8)$ & $54(68.3)$ & \multirow{2}{*}{ n.s. } \\
\hline Skin involvement, N (\%) & $6(5.7)$ & $2(7.7)$ & $4(5.1)$ & \\
\hline Arthritis, N (\%) & $12(11.4)$ & $5(19.2)$ & $7(8.9)$ & 0.014 \\
\hline Haematological involvement, N (\%) & $5(4.7)$ & $2(7.7)$ & $3(3.9)$ & \multirow{4}{*}{ n.s } \\
\hline Neuropsychiatric involvement, N (\%) & $3(2.9)$ & $1(3.9)$ & $2(2.5)$ & \\
\hline Vasculitis, N (\%) & $3(2.9)$ & 0 & $3(3.7)$ & \\
\hline Multisystemic involvement, N (\%) & $8(7.6)$ & $2(7.7)$ & $6(7.7)$ & \\
\hline \multicolumn{5}{|l|}{ Type of IS discontinued } \\
\hline Mycophenolate, N (\%) & $48(45.7)$ & $10(38.4)$ & $38(48.1)$ & n.s. \\
\hline Azathioprine, N (\%) & $30(28.6)$ & $4(15.4)$ & $26(32.9)$ & 0.03 \\
\hline Methotrexate, N (\%) & $15(13.3)$ & $8(30.7)$ & $6(7.6)$ & $<0.03 *$ \\
\hline Cyclosporine, N (\%) & $7(6.7)$ & $3(11.5)$ & $4(5.0)$ & n.s. \\
\hline Cyclophosphamide, N (\%) & $6(5.7)$ & $1(3.9)$ & $5(6.3)$ & n.s. \\
\hline
\end{tabular}

SLE, systemic lupus erythematosus; SDI, SLICCIACR damage index; HCQ, hydroxychloroquine; IS, immunosuppressant; Multisystemic: involvement of more than 2 organs requiring IS therapy. ${ }^{*} \mathrm{P}$ value refers to the comparison azathioprine vs. methotrexate and azathioprine vs. cyclosporine 


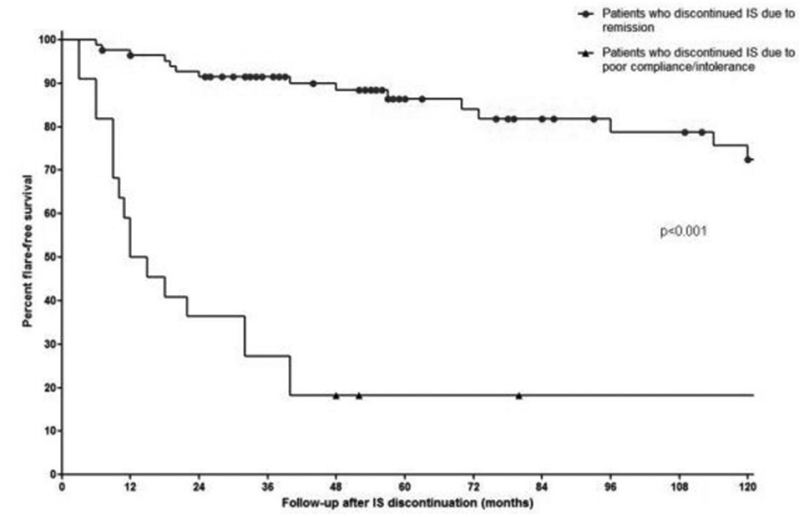

Abstract 173 Figure 1 Flare-free survival in patient who discontinued IS due to remission or due to poor compliance/intolerance along the follow-up after IS discontinuation

enable IS withdrawal, but no enough evidence is available regarding the benefits of IS discontinuation, especially in nonrenal SLE, or the risk of a subsequent flare. Our aims were to assess the rate of IS withdrawal in SLE remitted patients and the predictors of a subsequent flare and flare-free survival; moreover, to evaluate the effect of IS withdrawal on damage accrual. Methods We studied patients diagnosed with SLE between 1990 and 2018 treated with IS over their disease course who discontinued ISs. IS discontinuation was defined as complete withdrawal of any IS. Reasons for discontinuation were: remission, defined as clinical SLE Disease Activity Index (SLEDAI)$2 \mathrm{~K}=0$, or poor compliance/intolerance. Flares were defined according to SLEDAI Flare Index. Predictors of a subsequent flare and flare-free survival were analyzed by multivariate logistic regression and Cox regression analysis, respectively.

Results Eligible patients ever treated with IS were 319 out of 456. 139 patients discontinued ISs, 105 of them (75.5\%) due to remission. Mean \pm SD follow-up after IS withdrawal was 91 \pm 71 months (range 6-372).

26/105 remitted (24.7\%) and 23/34 unremitted patients $(67.6 \%)$ experienced a flare $(\mathrm{p}<0.001)$, a median (range) of 57 (6-264) and 8 months (1-72) after IS discontinuation, respectively $(p=0.009)$. Flare-free survival is depicted in figure 1 .

Table 1 reports characteristics of remitted patients, overall and according to flare occurrence after IS discontinuation. At multivariate logistic regression analysis, antimalarial intake (OR, 95\% CI 0.103, 0.023-0.452, $\mathrm{p}=0.003$ ), older age (OR 0.943, 0.892-0.997, $\mathrm{p}=0.039$ ) and the duration of remission before IS discontinuation (OR 0.943, 0.938-0.996, $\mathrm{p}=0.025$ ) were independent protective factors against flares after IS discontinuation. Conversely, no predictive factors of a longer time-to-flare were identified by multivariate Cox-regression analysis.

Median damage accrual was similar in patients who discontinued (1, range $0-8)$ or not IS (1, range $0-9)$ after adjusting for age, disease duration and cumulative prednisone dose.

We separately analyzed data of non-renal remitted patients (37 patients, $35.2 \%)$. The only variable associated with a lower flare rate was antimalarial intake (OR 0.048, 0.005-0.503, $\mathrm{p}=0.002$ ).

Conclusions In our cohort, one third of patients treated with IS discontinued the drug during the follow-up, in most cases due to remission. The use of antimalarials after IS discontinuation was independently associated with a significant decrease in the risk of flare.
Funding Source(s): None

Table 1 reports characteristics of remitted patients, overall and according to flare occurrence after IS discontinuation.

\section{SLE RESPONDER INDEX (SRI) UNDERESTIMATES CLINICAL RESPONSE IN MUSCULOSKELETAL SYSTEMIC LUPUS ERYTHEMATOSUS}

${ }^{1}$ Khaled Mahmoud, ${ }^{2}$ Ahmed Zayat, ${ }^{1}$ Md Yuzaiful Md Yusof, ${ }^{1}$ Elizabeth Hensor, ${ }^{1}$ Philip Conaghan, ${ }^{1}$ Paul Emery, ${ }^{1}$ Edward Vital*. ${ }^{1}$ University of Leeds; ${ }^{2}$ Bradford Teaching Hospitals NHS Foundation Trust

\subsection{6/lupus-2019-Ism.174}

Background Musuloskeletal (MSK) manifestations are common in SLE. Many recent clinical trials were negative or had small benefits vs. placebo. SRI is a common primary endpoint but has not been independently validated. Ultrasound is an objective measure of synovitis validated in inflammatory arthritis. We previously reported that in patients with inflammatory symptoms, 38\% had swollen joints, 27\% had subclinical inflammation (abnormal US but no clinically swollen joints) and $35 \%$ had no clinical or US inflammation. We also showed that subclinical tenosynovitis and PD were associated with significantly higher IgG, physician visual analogue score, tender joint count (Zayat et al. Rheumatology 2018). The purpose of the present study was to use ultrasound as a gold standard to evaluate clinical outcome measures of MSK lupus.

Methods A prospective pilot study was conducted in consecutive SLE patients with inflammatory musculoskeletal symptoms. Clinical assessments including SLEDAI-2K, BILAG-2004, 28-tender and swollen joint counts, physician and patient VAS and ultrasound were performed at 0,2 and 4 weeks following $120 \mathrm{mg}$ intramuscular methylprednisolone acetate. Responsiveness was analysed using changes and effect sizes using Cohens criteria.

Results 20 patients were recruited. 15/20 had clinical swelling at baseline. The others had abnormal ultrasound and/or early morning stiffness and tenderness. All clinical and US parameters were significantly improved at week 4 (all p0.01). Musculoskeletal-BILAG score improved in 16/20. MusculoskeletalSLEDAI improved in $7 / 20$. SRI-4 criteria were assessed in 19 patients with SLEDAI $\geq 4$ at baseline met in $9 / 19$ at 4 weeks. Effect sizes at 4 weeks were large $(>0.5)$ for US (power Doppler and Greyscale), physician VAS and BILAG and medium $(>0.3)$ for joint counts and SLEDAI. Large effect sizes for improvement in US greyscale and power Doppler were observed in both SRI responders $(\mathrm{r}=-0.51$ and -0.56 respectively) and non-responders $(\mathrm{r}=-0.62$ and -0.59$)$ at 4 weeks. In SRI non-responders swollen joint counts improved by $20 \%$, $50 \%$ and $70 \%$ in $7 / 10,7 / 10$ and $6 / 10$ patients respectively.

Conclusions This is the first study to measure the responsiveness of clinical outcome measures in musculoskeletal SLE against an objective inflammation measure. BILAG-2004 and physician VAS were the most responsive clinical instruments. US was highly responsive in musculoskeletal SLE, while SLEDAI-2K and joint counts appeared suboptimal for detection of improvement. These results suggest that clinical trials based on the SLEDAI-2K and SRI-4 may underestimate the efficacy of therapy in SLE.

Funding Source(s): LupusUK, NIHR 